\title{
Relationship between Lower Limb Angular Kinematic Variables and the Effectiveness of Sprinting during the Acceleration Phase
}

\author{
Artur Struzik, ${ }^{1}$ Grzegorz Konieczny, ${ }^{2}$ Mateusz Stawarz, ${ }^{3}$ Kamila Grzesik, ${ }^{3}$ \\ Slawomir Winiarski, ${ }^{4}$ and Andrzej Rokita ${ }^{1}$ \\ ${ }^{1}$ Department of Team Sport Games, University School of Physical Education, Mickiewicza 58 Street, 51-684 Wrocław, Poland \\ ${ }^{2}$ Faculty of Health Sciences and Physical Education, Witelon State University of Applied Sciences, \\ Sejmowa 5A Street, 59-220 Legnica, Poland \\ ${ }^{3}$ University School of Physical Education, Paderewskiego 35 Avenue, 51-612 Wrocław, Poland \\ ${ }^{4}$ Department of Biomechanics, University School of Physical Education, Mickiewicza 58 Street, 51-684 Wrocław, Poland
}

Correspondence should be addressed to Artur Struzik; artur.struzik@awf.wroc.pl

Received 15 April 2016; Accepted 23 June 2016

Academic Editor: Alberto Borboni

Copyright (C) 2016 Artur Struzik et al. This is an open access article distributed under the Creative Commons Attribution License, which permits unrestricted use, distribution, and reproduction in any medium, provided the original work is properly cited.

\begin{abstract}
The ability to reach a high running velocity over a short distance is essential to a high playing performance in team games. The aim of this study was to determine the relationship between running time over a 10-meter section of a 30-meter sprint along a straight line and changes in the angle and angular velocity that were observed in the ankle, knee, and hip joints. The possible presence may help to optimize motion efficiency during acceleration sprint phase. Eighteen girls involved in team sports were examined in the study. The Fusion Smart Speed System was employed for running time measurements. The kinematic data were recorded using the Noraxon MyoMotion system. Statistically significant relationships were found between running time over a 10-meter section and the kinematic variables of hip and ankle joints. An excessively large flexion in hip joints might have an unfavorable effect on running time during the acceleration phase. Furthermore, in order to minimize running time during the acceleration phase, stride should be maintained along a line (a straight line) rather than from side to side. It is also necessary to ensure an adequate range of motion in the hip and ankle joints with respect to the sagittal axis.
\end{abstract}

\section{Introduction}

A 30-meter run is a popular test used by coaches in team sports in order to evaluate the level of speed abilities in athletes $[1,2]$. Over such a distance, consecutive phases of a sprint can be distinguished in athletes from team sports: start, push-off, acceleration, and, contrary to sprinters, maximum velocity phase $[1,3-6]$. The acceleration phase for these athletes is much shorter than that for track sprinters, and the maximal running velocity is reached earlier $[1,3,5,6]$. However, the time of a 30-meter run should be interpreted carefully due to the substantial time differences within the test distance. For example, two athletes can have a similar running time over a 30-meter distance. However, the split times in individual sections of the run may differ significantly. Therefore, the individual sprinting workout of a player from team sports should be based on split time recordings $[2,3]$.
Running at a substantial velocity is observed among soccer players at 70-90-second intervals $[2,7,8]$. However, team sport players never reach maximal velocity during a sprint under actual game conditions $[1,6,9]$. Of all the sprints that are performed by soccer players, $49 \%$ are shorter than $10 \mathrm{~m}$ and $96 \%$ are shorter than $30 \mathrm{~m}[2,7]$. Therefore, the ability to reach high running velocity over a short distance (acceleration) is essential to high playing performance $[1,3,6$, $8-16]$. Consequently, coaches should plan running workouts for team sport athletes that focus on the acceleration phase rather than maximum velocity $[1,6,9]$. The time it takes an athlete to run a 10 -meter section is considered a measure of acceleration ability, and the distance of 10 meters is considered a conventional distance of acceleration (its initial part, with velocity increment being the highest) $[6,8,10,11$, $13,14,17,18]$. 
Each of the sprinting phases is characterized by different technical and physiological demands to maximize motion efficiency. Therefore, different training programs should be designed to improve the individual phases of a sprint [3, $4,13,19]$. The specific nature of each sport should also be considered because running technique used by athletes from team sports differs greatly from the technique that is used by track athletes $[1,3,6,20]$. Team sport athletes run with a relatively lower height of the center of mass, less knee flexion during swing phase, and lower knee lift $[1,20]$. This type of running helps team sport athletes to change their direction of movement faster [20].

The major goal of a team sport player when running over a short distance is to cover the distance as soon as possible in order to be ahead of either the offensive or defensive actions of the opponents. Therefore, a maximization of the horizontal component of velocity of the athlete's center of mass is needed. Running velocity is represented by a mathematical product of stride length and stride frequency. In order to maximize running velocity, it is necessary to proportionally increase both variables. Furthermore, each running phase requires different length-to-frequency ratios in order to maximize the effectiveness $[9,11,12,14,19,21$, 22].

Acceleration ability can be improved by emphasizing the kinematic variables (which characterize the running technique) and/or kinetic variables (associated with the effect of force) during training, considering their mutual relations. The running technique should become the basis on which other variables, which are responsible for energy production or muscle strength, can be developed $[9,14$, 15]. For example, a player with a greater potential for the development of high running velocity will lose to opponents due to a longer reaction time and poorer starting technique [23]. Teaching proper movement technique is essential in young athletes, with their training being more general and unfocused towards the development of strength abilities. During the acceleration phase, running technique should be most conducive to developing high speed in a short time [15]. Therefore, adequate synchronization of movements of the upper limbs with respect to the lower limbs is needed [22].

However, researchers do not agree on the most effective training method to improve acceleration ability and on which variables should be improved $[9,12,14,16,17]$. This is likely caused by an unclear contribution of individual joints to running performance [24]. Mann and Herman [21] reported that a lower value of knee extension at take-off positively affects sprinters' running times. Analogous conclusions were drawn by Murphy et al. [11] regarding the acceleration phase. Furthermore, Krell and Stefanyshyn [24] found that sprinters who had shorter running times were characterized by higher maximal rates of metatarsophalangeal extension. Ansari et al. [25] suggested that kinematic variables, such as knee angle, hip angle, ankle angle, shoulder rotation, and extension, are of key importance to the sprinting technique and have a clear effect on sprint performance. In contrast, Ranjan [26] found nonsignificant relationships between the angles in the knee, hip, and ankle joint and the sprint start performance. Lockie et al. [27] also did not find statistically significant relationships between the $0-5$ and $0-10 \mathrm{~m}$ sprint times and the angles in the knee, hip, and ankle joints.

A substantial number of accelerations and cuts over short distances resulting from changes in the direction of movement make the ability to reach a high running velocity over a short distance essential for high playing performance $[1,3,6,8-16]$. However, the vast majority of studies on relationships between kinematic variables and running time have evaluated populations of runners $[4,17,21,22,24-$ 26]. Furthermore, reports concerning the running technique of young athletes from team sports are also scarce [5]. The aim of this study was to determine the relationship between the running time over a 10 -meter section of a 30-meter sprint along a straight line and changes in the angle and angular velocity in the ankle, knee, and hip joints. Therefore, the authors attempted to answer the question of whether a technique of lower limb movement during the sprint acceleration phase significantly correlates with the running time over this section.

\section{Materials and Methods}

Examinations involving members of the Lower Silesian Voivodeship Team were carried out in a group of 265 participants selected from young people who trained in team sports (soccer, basketball, volleyball, and handball). Eighteen girls with the highest potential speed abilities were selected from this group. This selection was based on the maximum height of the countermovement jump [28]. The study group was characterized by the following mean values $( \pm S D)$ : body height, $165.5 \pm 10.5 \mathrm{~cm}$; body mass, $52.4 \pm 10.3 \mathrm{~kg}$; and age, $13.7 \pm 1.4$ years. Training experience was $4.2 \pm 1.7$ years. Parents were informed about the purpose of the study and provided written permission for the tests. The experiments were carried out in the Ball Games Research Laboratory (with PN-EN ISO 9001:2009 certification). The research project was approved by the Senate's Research Bioethics Commission, and the procedure complied with the Declaration of Helsinki regarding human experimentation.

A fifteen-minute warm-up was administered before the measurements. The athletes performed a trial test before the measurement. Each participant performed two 30-meter runs over a straight line. The analysis was based on the trial with the shorter time of the first 10-meter section obtained by each participant. The distance to be run was purposively elongated in order to avoid the slow-down effect in the final part of the run [10].

The Fusion Smart Speed System (Fusion Sport, Coopers Plains, QLD, Australia) was used to measure the sprint time over individual sections. This system is comprised of gates, with each gate equipped in an infrared transmitter and a light reflector. A distance of 2 meters was adjusted between the infrared transmitter and the light reflector. Individual gates were spaced 5 meters (beginning from the start line) apart to record the time of crossing the infrared radiation beam. The gate at the distance of 30 meters denoted the finish line. The split times at distances of 5, 10, 15, 20, and $25 \mathrm{~m}$ were also recorded. 


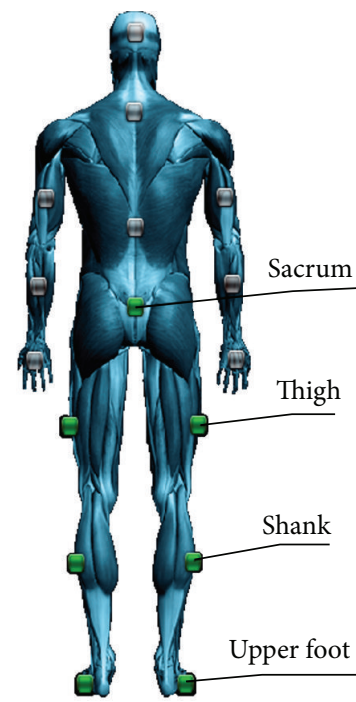

FIGURE 1: The places of deployment of inertial sensors (based on 16joint-segment rigid-body model from MR3 software).

A Noraxon MyoMotion (Scottsdale, AZ, USA) motion analysis system was used to analyze the kinematic variables. A small inertial measurement unit (IMU) placed on a body segment tracks its 3D angular orientation. By placing individual IMU sensors on two neighbouring body segments, one can evaluate the range of motion in the joint placed between these segments. This principle might be extended from an individual movement of a joint over simultaneous measurement of the motion of the whole body in individual major joints. The MyoMotion sensors transmit the motion of the human body directly to the MyoMotion receiver to quantify the angular changes of the selected body segments. The information from a 3D accelerometer, gyroscope, and magnetometer is used to measure 3D rotation angles of each sensor in absolute space. The system is entirely wireless and does not need calibration of the measurement space, which allows for measurements outside the laboratory. System is entirely independent of external cameras. MyoMotion Research inertial sensors were placed according to the rigidbody model, with 16 joint segments used in the MR3 software on shoes (top of the upper foot, slightly below the ankle), shanks (frontal on the tibia bone), thighs (frontal attachment to the lower quadrant of the quadriceps, slightly above the knee cap, area of lowest muscle belly displacement in motion), and bony area of the sacrum (Figure 1). The sensors were attached with special fixation straps (for pelvis) and elastic straps. Calibration was carried out using the upright position in order to determine the value of the $0^{\circ}$ angle in the studied joints. The sampling frequency for the inertial sensors was set at $200 \mathrm{~Hz}$. Instantaneous changes in the joint angles in the area of the lower limb were recorded: hip joint (with respect to the long, transverse, and sagittal axes), knee joint (with respect to the transverse axis), and ankle joint (with respect to the long, transverse, and sagittal axes) during a 30-meter run along a straight line. Positive values of the angle depending on the joint and axis correspond to flexion, abduction, external rotation, dorsiflexion, and inversion. The angular velocities in the area of these joints were determined based on the derivative of the distance (angle) over time [29].

The participants started from a standing position at the light signal. The left lower limb of a study participant was placed ahead of the start line so that the first step was performed with the right limb. Three entire running gait cycles were used for both the left and right lower limbs (steps 3 to 8 occurring during the acceleration phase), determined based on the instantaneous angle in the knee joint (the border between the cycles was the minimum value of the angle). Manual recognition was used to isolate the running gait cycles. The running steps were counted at a distance of $10 \mathrm{~m}$, adopted as a conventional acceleration phase $[6,13,18]$. The first two running steps were neglected in the analysis because they started at the near-zero velocity, thus being substantially different from the other six.

Three entire gait cycles (as a whole) were normalized with respect to time and averaged for each lower limb separately. Then, the mean angle and angular velocity profiles were calculated with standard deviations. Before generating the graphical presentation shown in Figures 2 and 3, the profiles were smoothed. The procedure of standardization (normalization of variables) allows for intercomparison, even if the subjects have differed in terms of somatic parameters. Therefore, the curve shapes were normalized with respect to time (relative time expressed as a percentage).

In order to analyze the relationships between the sprint time over the section of $10 \mathrm{~m}$ and the individual kinematic variables, Spearman's rank correlation coefficient was used due to the lack of normal distribution of the studied variables. The level of significance was set at $\alpha=0.05$. Furthermore, multiple regression was used to find the variable that was the most correlated with running time.

\section{Results}

Figure 2 presents the instantaneous changes in the angle in the area of lower limb joints: hip joint (with respect to the long, transverse, and sagittal axes), knee joint (with respect to the transverse axis), and ankle joint (with respect to the long, transverse, and sagittal axes) during the acceleration phase of a 30-meter run along a straight line. In Figure 2, steps 3, 5, and 7 (from the start line) for the right lower limb and steps 4,6 , and 8 (from the start line) for the left lower limb were presented. Analogously, changes in the variable of angular velocity are presented in Figure 3.

Statistically significant correlations between running time over a 10-meter section and individual kinematic variables of the hip joint were found for the maximum flexion angle, flexion-extension range of angular velocity, maximum adduction angle, maximum adduction angular velocity, maximum abduction angular velocity, abduction-adduction range of motion, and abduction-adduction range of angular velocity. Furthermore, statistically significant correlations between running time over a 10-meter section and individual kinematic variables of the ankle joint occurred for maximum eversion angular velocity, inversion-eversion range of angular velocity, maximum dorsiflexion angle, maximum plantar 

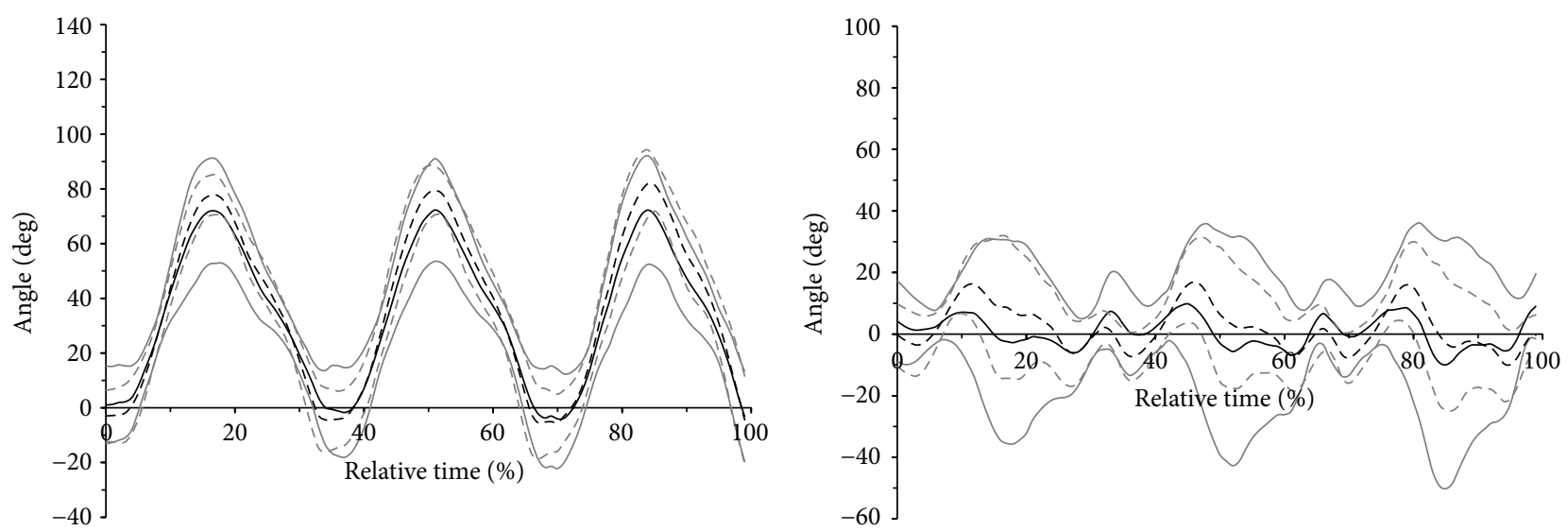

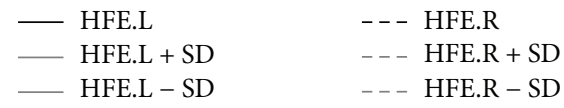

(a) Hip flexion-extension angle
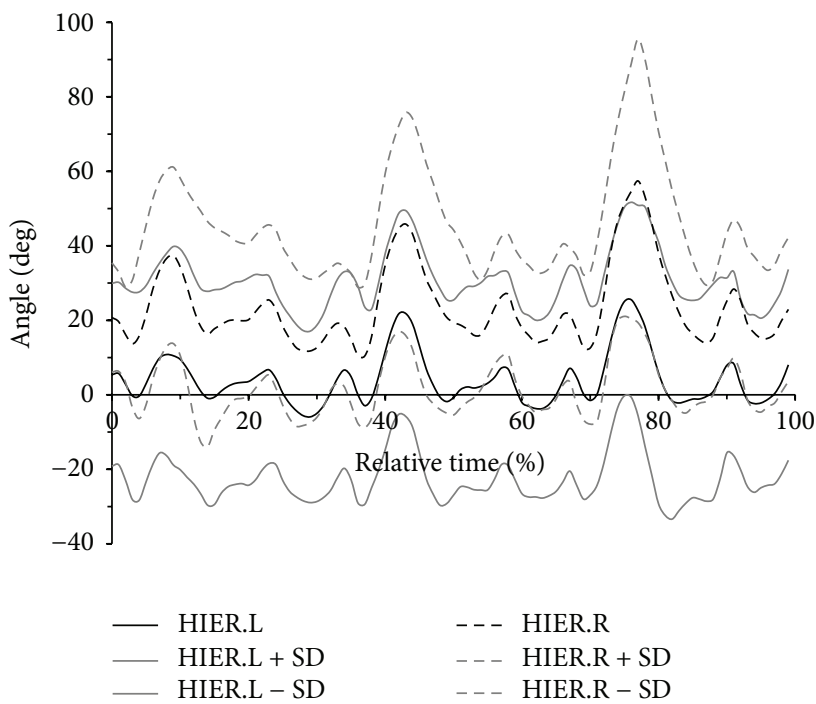

(c) Hip internal-external rotation angle

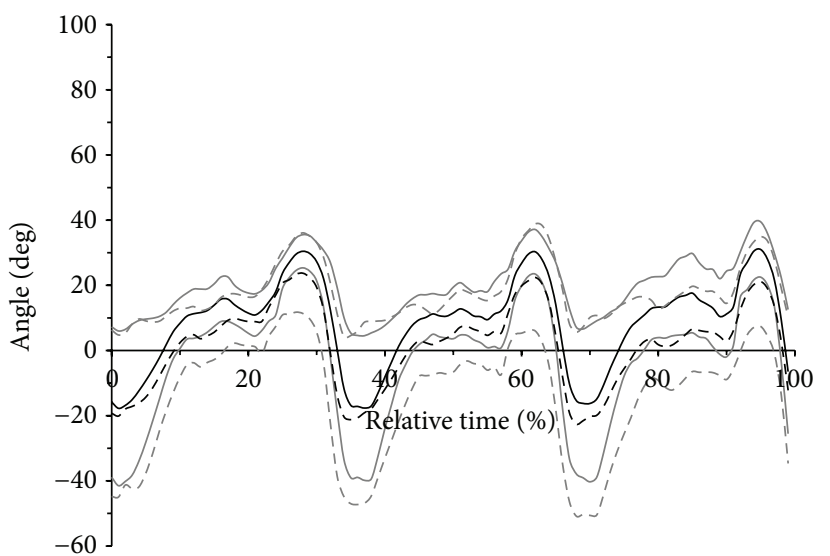

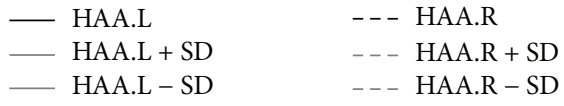

(b) Hip abduction-adduction angle
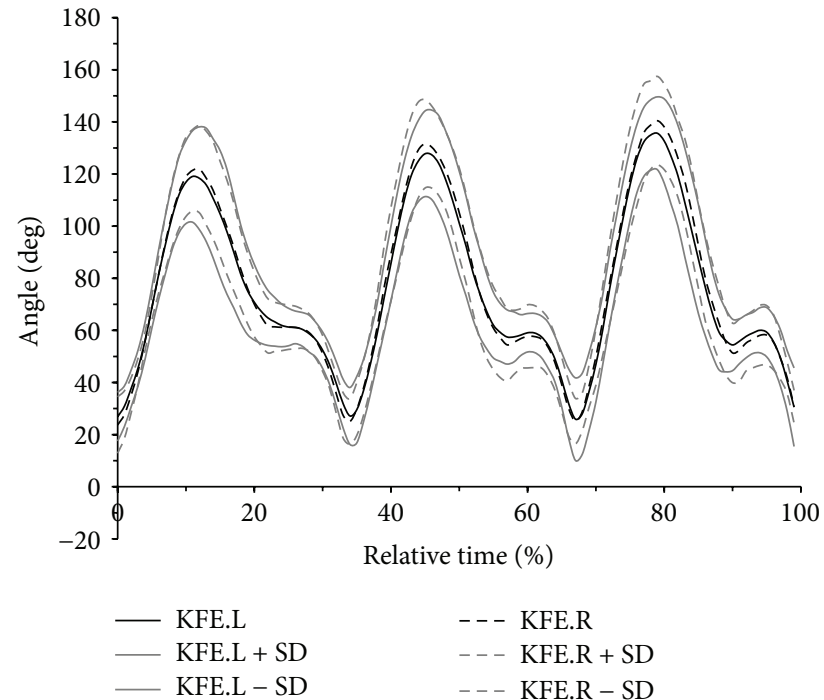

(d) Knee flexion-extension angle
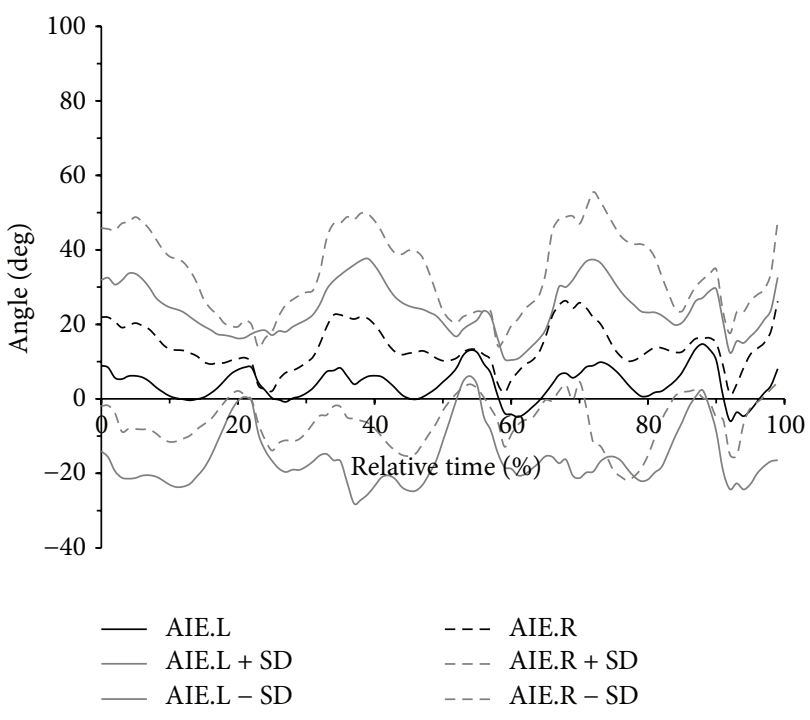

(f) Ankle inversion-eversion angle

Figure 2: Continued. 


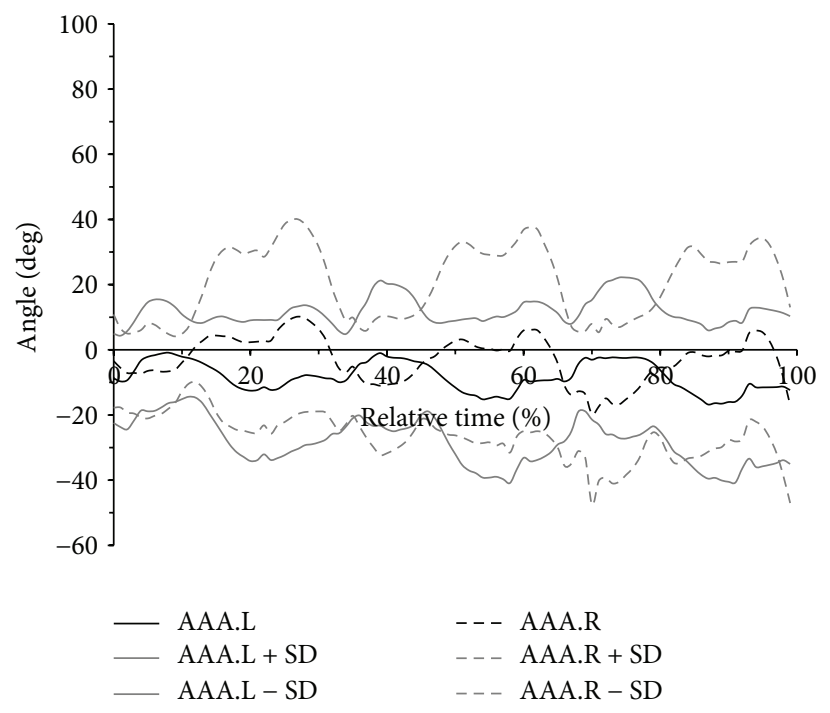

(g) Ankle abduction-adduction angle

FIGURE 2: Instantaneous changes in the angle in the area of lower limb joints with respect to specific axes during a 30-meter sprint acceleration phase along a straight line for the left limb (continuous line) and right limb (dashed line).

flexion angle, maximum abduction angle, maximum abduction angular velocity, maximum adduction angle, maximum adduction angular velocity, abduction-adduction range of motion, and abduction-adduction range of angular velocity. For other recorded variables of the hip joint and ankle joint, the relationships with running time over a 10-meter section were not statistically significant. These correlations differed in their magnitude and significance depending on the running gait. Values of correlation coefficients for the hip joint are presented in Table 1, whereas these values for the ankle joint are shown in Table 2. No statistically significant correlations were found between running time over a 10meter section and individual kinematic variables of the knee joint. Multiple regression analysis revealed that the most significant correlation with running time was observed for the maximum hip flexion angle during the eighth running step.

\section{Discussion}

Statistically significant relationships between running time over a 10-meter section and kinematic variables of the lower limbs occurred most often for one of the body sides, mainly left (Tables 1 and 2). This observation might suggest that the movement pattern during the initial acceleration phase is somehow determined by the asymmetric start (the first step performed by the right lower limb). Therefore, despite alternate work of the limbs, the movement kinematics in the acceleration phase were not as symmetrical as in the maximal velocity phase [29]. Coaches should therefore focus on improving the acceleration phase and on starting from both the left and right legs (at zero initial velocity and at flying start). This improvement is associated with dynamically changing situations on the field or court, which cause team sport athletes to perform many explosive movements during
TABLE 1: Values of correlation coefficients $(r)$ between running time over a 10-meter section and individual kinematic variables of the hip joint.

\begin{tabular}{lcc}
\hline Variable & $\begin{array}{c}\text { Running } \\
\text { step }\end{array}$ & $r$ \\
\hline Maximum flexion angle & 4 & $0.47^{*}$ \\
\hline Flexion-extension range of & 8 & $0.56^{*}$ \\
angular velocity & 4 & $-0.48^{*}$ \\
\hline & 4 & $-0.60^{*}$ \\
Maximum adduction angle & 6 & $-0.65^{*}$ \\
& 8 & $-0.67^{*}$ \\
\hline Maximum adduction & 6 & $-0.47^{*}$ \\
angular velocity & 8 & $-0.64^{*}$ \\
\hline Maximum abduction & 8 & $-0.52^{*}$ \\
angular velocity & 4 & $-0.53^{*}$ \\
\hline Abduction-adduction & 6 & $-0.53^{*}$ \\
range of motion & 8 & $-0.66^{*}$ \\
\hline Abduction-adduction & 6 & $-0.47^{*}$ \\
range of angular velocity & 8 & $-0.68^{*}$ \\
\hline
\end{tabular}

${ }^{*}$ Statistically significant at $p<0.05$.

the game, such as rapid starts, accelerations and cuts, changes in the direction of movement, or jumps. For instance, soccer players perform 150-250 brief intense actions lasting 1.9-2.7 s during a match [7]. The effectiveness of the above movements depends mainly on lower limb power $[1,2,13,19]$.

Lockie et al. [14, 15] argue that the beneficial effect on running time over a 10 -meter section is from greater stride length, longer flight time, and shorter contact time. 

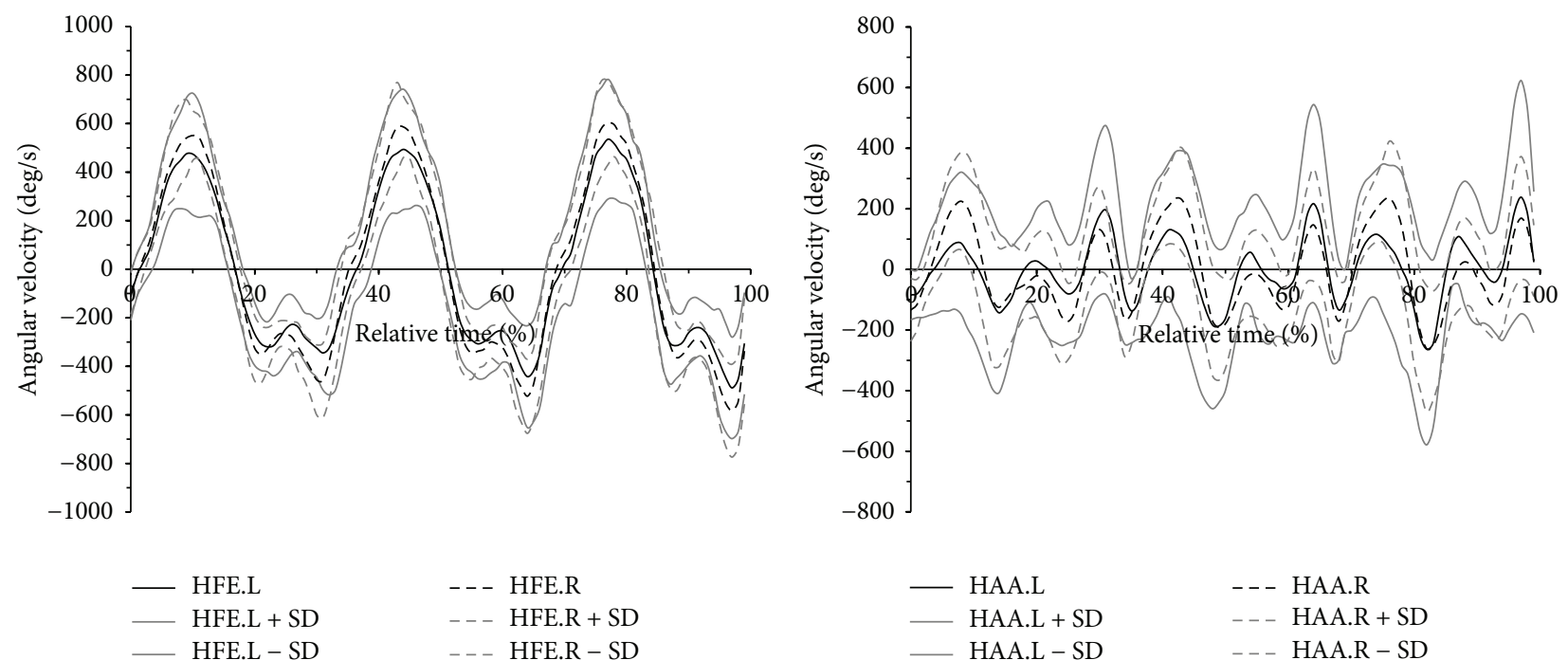

(b) Hip abduction-adduction angular velocity
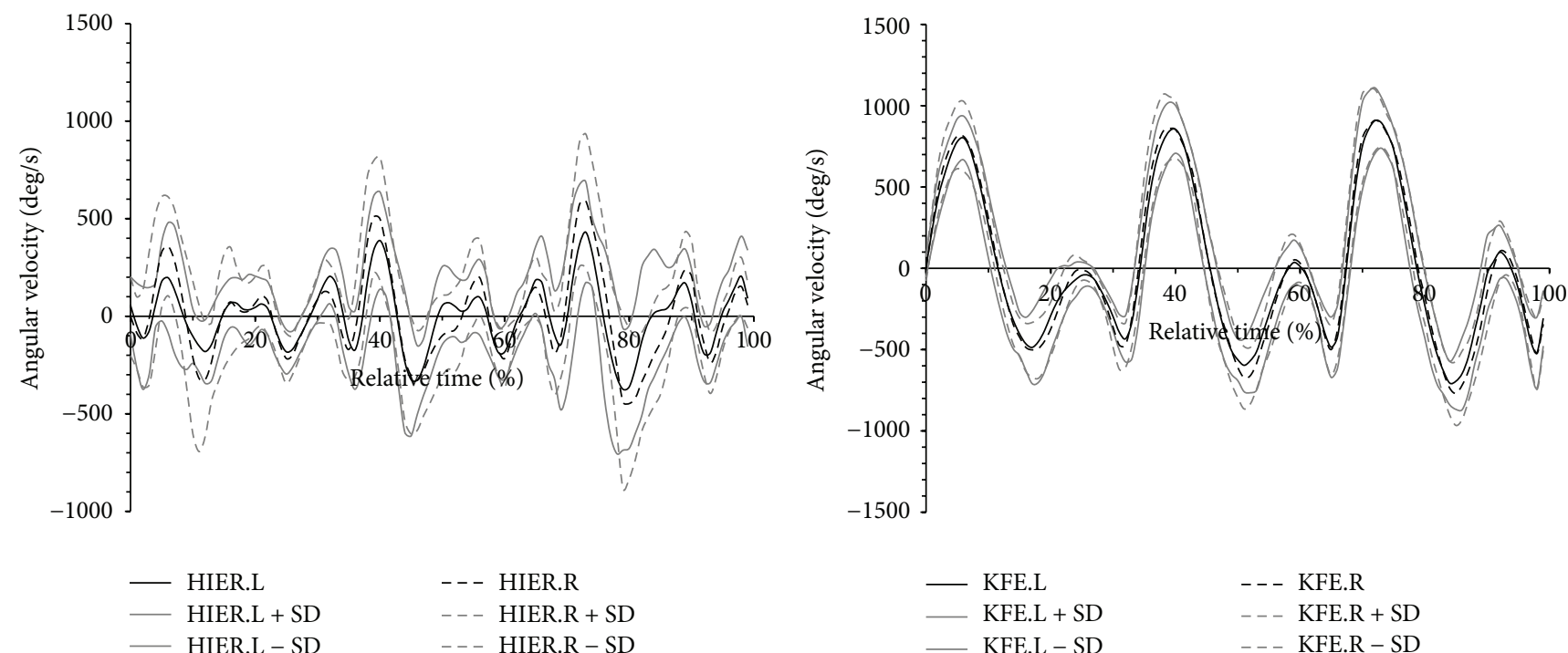

(c) Hip internal-external rotation angular velocity

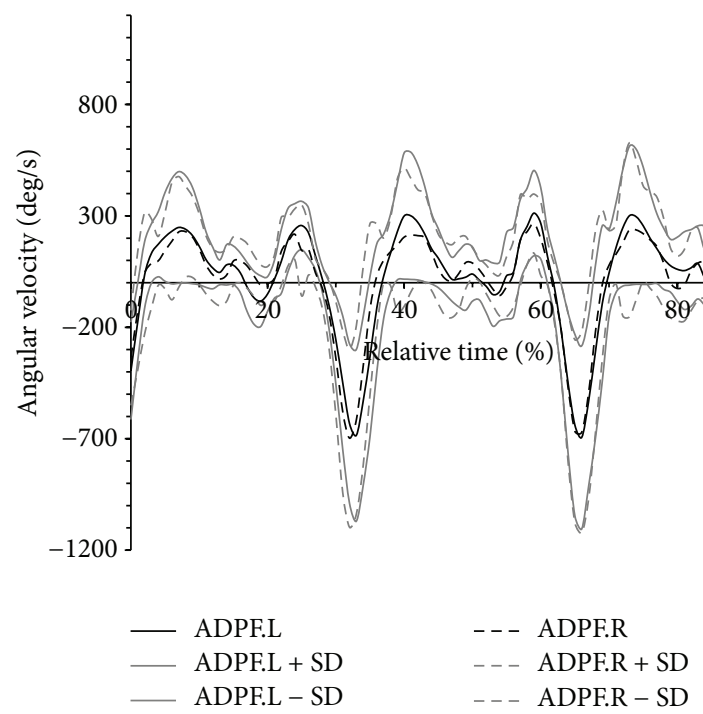

(e) Ankle dorsi-plantarflexion angular velocity 


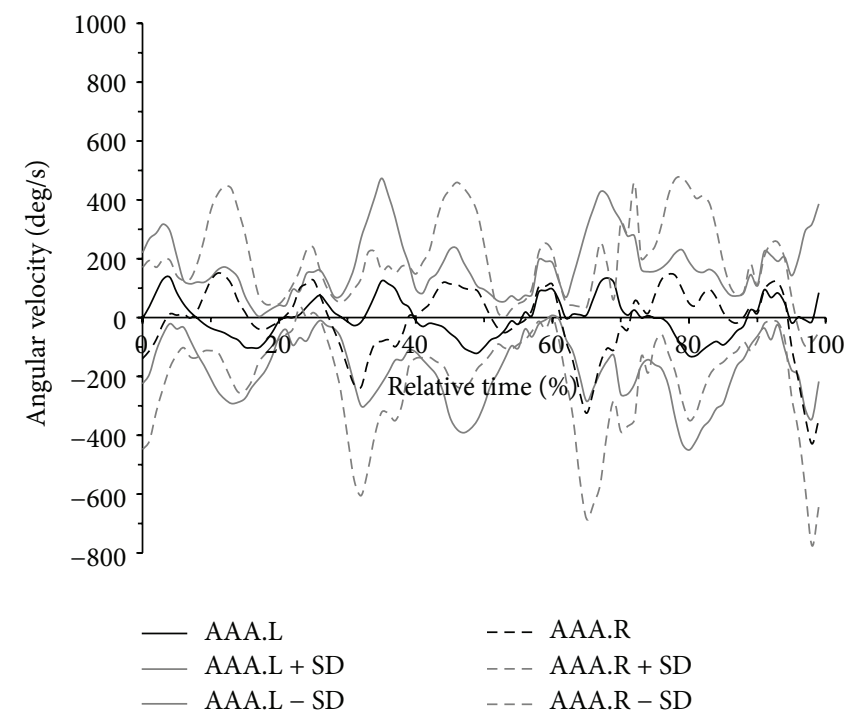

(g) Ankle abduction-adduction angular velocity

FiguRE 3: Instantaneous changes in the angular velocity in the area of lower limb joints with respect to specific axes during a 30-meter sprint acceleration phase along a straight line for the left limb (continuous line) and right limb (dashed line). Continuous or dashed thin grey lines were denoted \pm SD for the left and right limb, respectively.

TABLE 2: Values of correlation coefficients $(r)$ between running time over a 10 -meter section and individual kinematic variables of the ankle joint.

\begin{tabular}{lcc}
\hline Variable & $\begin{array}{c}\text { Running } \\
\text { step }\end{array}$ & $r$ \\
\hline $\begin{array}{l}\text { Maximum eversion angular } \\
\text { velocity }\end{array}$ & 8 & $-0.50^{*}$ \\
\hline $\begin{array}{l}\text { Inversion-eversion range of } \\
\text { angular velocity }\end{array}$ & 8 & $-0.47^{*}$ \\
\hline Maximum dorsiflexion angle & 3 & $0.64^{*}$ \\
\hline Maximum plantar flexion angle & 5 & $-0.49^{*}$ \\
\hline Maximum abduction angle & 5 & $0.48^{*}$ \\
\hline Maximum abduction angular & 4 & $-0.60^{*}$ \\
velocity & 6 & $-0.47^{*}$ \\
\hline Maximum adduction angle & 6 & $-0.59^{*}$ \\
\hline Maximum adduction angular & 8 & $-0.65^{*}$ \\
velocity & 4 & $-0.67^{*}$ \\
\hline $\begin{array}{l}\text { Abduction-adduction range of } \\
\text { motion }\end{array}$ & 4 & $-0.74^{*}$ \\
\hline Abduction-adduction range of & 6 & $-0.56^{*}$ \\
angular velocity & 8 & $-0.55^{*}$ \\
\hline S & 4 & $-0.75^{*}$ \\
\hline
\end{tabular}

${ }^{*}$ Statistically significant at $p<0.05$.

However, the statistically significant positive correlations between running time over a 10-meter section and maximal hip flexion angle (steps 4 and 8) suggest that excessively large flexion in hip joints is unfavorable for running time during the acceleration phase. Furthermore, the maximum hip flexion angle during the eighth running step showed the highest relationship with running time among all of the studied variables. These relationships may suggest that the stride length during the acceleration phase should not be too long. This result is likely to be supported by the lack of statistically significant relationships between the running time over a 10-meter section and kinematic variables for the knee joint with respect to the transverse axis. Statistically significant relationships between the running time over a 10meter section and the dorsiflexion angle (positive, step 3) and plantar flexion angle (negative, step 5) appear to support this thesis. Therefore, it seems that the running stride should be performed dynamically (shorter contact time) rather than strongly forward (adequate proportion of stride length to stride frequency). This result appears to be supported by a statistically significant negative relationship between running time over a 10-meter section and the hip flexion-extension range of angular velocity (step 4). Indeed, some authors have documented a beneficial effect of stride frequency on the effectiveness of the acceleration phase $[9,11]$. Therefore, in order to improve the acceleration phase, coaches should consider exercises that increase power in the lower limbs. The running stride during a sprint can be regarded as a ballistic movement due to the duration of the phase of foot contact with the ground being shorter than $0.2 \mathrm{~s}[9,13-$ $16,19]$. The athletes studied by Lockie et al. [14, 15] were much older ( $22.6 \pm 3.2$ years) than the athletes included in our study, which may justify the differences. However, the step frequency is more difficult to improve than the step length during the training process. Additionally, faster athletes employ a higher step frequency with a similar step length to those of their slower counterparts [17]. It has been suggested that the angle between the thigh and body trunk during the acceleration phase should be approximately $90^{\circ}$ 
[16]. However, the athletes who participated in our study obtained the following mean values of maximal hip flexion: $78.3 \pm 7.1^{\circ}$ (third step), $74.1 \pm 16.5^{\circ}$ (fourth step), $81.7 \pm 8.2^{\circ}$ (fifth step), $75.1 \pm 16.8^{\circ}$ ( sixth step), $83.8 \pm 10.4^{\circ}$ (seventh step), and $75.8 \pm 17.3^{\circ}$ (eighth step). Therefore, the above values were slightly smaller than those suggested by Spinks et al. [16].

Statistically significant negative relationships between running time over a 10 -meter section and the maximal adduction angle for the hip joint (steps 4, 6, and 8) and ankle joint (steps 6 and 8) may demonstrate that the running gait should be performed in a line consistent with the direction of running (straight line) rather than from side to side. This result appears to be supported by a statistically significant positive relationship between running time over a 10-meter section and the maximum abduction angle in the ankle joint (step 5). An efficient sprint involves placing the foot closer to the location of the vertical projection (on the ground) of the center of gravity of the runner's body. In order to counteract the horizontal deceleration, the foot, after contacting the ground, should move towards the rear with respect to the general center of gravity with the horizontal velocity, which is at least equal compared to the velocity of the general center of gravity of the running person [21]. Statistically significant negative relationships between the running time over a 10meter section and the maximal values and range of angular velocity with respect to the sagittal axis of the hip joint and ankle joint and with respect to the long axis of the ankle joint (Tables 1 and 2) seem to support the need for performing the running gait as dynamically as possible.

Ansari et al. [25] suggest that kinematic variables, such as the hip angle, knee angle, and ankle angle, are essential to the sprinting technique. In contrast, Ranjan [26] and Lockie et al. [27] did not find statistically significant relationships between the angles in the knee, hip, and ankle joints and the sprint performance. In the present study, only the kinematic variables of the hip joint and ankle joint showed a significant relationships with the running time over a 10-meter section of a 30-meter sprint. Theoretically, a greater range of motion and knee flexion angle should also positively affect sprint performance. During the recovery portion of the stride, the heel should remain as close to the hips (buttocks) as possible, which helps to shorten the inertia radius and achieve a higher angular velocity in the knee joint $[21,25,30]$. However, Mann and Herman [21] and Murphy et al. [11] have demonstrated that a lower value of knee extension at take-off positively affects the running time for sprinters, which might result from differences in the motion pattern that should occur in the acceleration phase and maximal velocity phase. However, no statistically significant relationships were found in our study between running time and the values of angles, angular velocities, and range of motion in the knee joint. A lack of these relationships may suggest that this part of the motion pattern was performed properly by the participants rather than the variables do not affect running time.

Statistically significant negative relationships were found between running time over a 10-meter section and the ranges of motion of the hip joint (steps 4, 6, and 8) and the ankle joint (steps 4,6 , and 8 ) in the sagittal plane. Therefore, in order to maximize the velocity (minimize time) of running during the acceleration phase, an adequate level of mobility with respect to the sagittal plane in the hip joint and the ankle joint is needed. A relatively high range of motion in the joints of the lower limb is necessary to obtain an adequate running stride [27].

\section{Conclusions}

This study contains technical running guidelines for young athletes that can be used during training. However, it should be noted that the relationships between running time over a 10-meter section of a 30-meter sprint and kinematic variables of lower limbs might differ depending on the age of the athletes, the sport, and the running technique level. Movement technique also depends on the running phase. The relationships between running time over a 10 -meter section of a 30-meter sprint and the kinematic variables of the lower limbs refer only to the technical aspect of the running gait, which does not consider (directly) the kinetic variables. Therefore, further studies should be extended by kinetic variables of the lower limbs and the search for relationships between kinetic and kinematic variables. The actual practical implications can be reduced to the three conclusions presented below:

(1) An excessively large flexion in hip joints might have an unfavorable effect on running time during the acceleration phase of a 30-meter sprint.

(2) In order to minimize running time during the acceleration phase, running should be maintained more in a line (a straight line) rather than from side to side.

(3) A beneficial relationship with running time during the acceleration phase was found for the range of motion in hip and ankle joints with respect to the sagittal axis.

\section{Competing Interests}

The authors declare that they have no competing interests.

\section{Acknowledgments}

This study was made possible by funds from the Polish Ministry of Science and Higher Education within the program "Development of Academic Sport 2013" (Grant no. RSA2 019 52).

\section{References}

[1] J. B. Cronin and K. T. Hansen, "Strength and power predictors of sports speed," Journal of Strength and Conditioning Research, vol. 19, no. 2, pp. 349-357, 2005.

[2] U. Wisløff, C. Castagna, J. Helgerud, R. Jones, and J. Hoff, "Strong correlation of maximal squat strength with sprint performance and vertical jump height in elite soccer players," British Journal of Sports Medicine, vol. 38, no. 3, pp. 285-288, 2004.

[3] M. J. Barr, J. M. Sheppard, T. J. Gabbett, and R. U. Newton, "Long-term training-induced changes in sprinting speed and 
sprint momentum in elite rugby union players," Journal of Strength and Conditioning Research, vol. 28, no. 10, pp. 27242731, 2014.

[4] L. Charalambous, G. Irwin, I. N. Bezodis, and D. Kerwin, "Lower limb joint kinetics and ankle joint stiffness in the sprint start push-off," Journal of Sports Sciences, vol. 30, no. 1, pp. 1-9, 2012.

[5] M. C. Rumpf, J. B. Cronin, J. L. Oliver, and M. Hughes, "Assessing youth sprint ability-methodological issues, reliability and performance data," Pediatric Exercise Science, vol. 23, no. 4, pp. 442-467, 2011.

[6] W. Young, B. McLean, and J. Ardagna, "Relationship between strength qualities and sprinting performance," The Journal of Sports Medicine and Physical Fitness, vol. 35, no. 1, pp. 13-19, 1995.

[7] T. Cotte and J.-C. Chatard, "Isokinetic strength and sprint times in English Premier League football players," Biology of Sport, vol. 28, no. 2, pp. 89-94, 2011.

[8] M. A. Newman, K. M. Tarpenning, and F. E. Marino, "Relationships between isokinetic knee strength, single-sprint performance, and repeated-sprint ability in football players," Journal of Strength and Conditioning Research, vol.18, no. 4, pp. 867-872, 2004.

[9] R. G. Lockie, A. J. Murphy, T. J. Knight, and X. A. K. Janse de Jonge, "Factors that differentiate acceleration ability in field sport athletes," Journal of Strength and Conditioning Research, vol. 25, no. 10, pp. 2704-2714, 2011.

[10] G. Moir, C. Button, M. Glaister, and M. H. Stone, "Influence of familiarization on the reliability of vertical jump and acceleration sprinting performance in physically active men," Journal of Strength and Conditioning Research, vol. 18, no. 2, pp. 276-280, 2004.

[11] A. J. Murphy, R. G. Lockie, and A. J. Coutts, "Kinematic determinants of early acceleration in field sport athletes," Journal of Sports Science and Medicine, vol. 2, no. 4, pp. 144-150, 2003.

[12] G. D. Myer, K. R. Ford, J. L. Brent, J. G. Divine, and T. E. Hewett, "Predictors of sprint start speed: the effects of resistive groundbased vs. inclined treadmill training," Journal of Strength and Conditioning Research, vol. 21, no. 3, pp. 831-836, 2007.

[13] R. G. Lockie, A. J. Murphy, A. B. Schultz, T. J. Knight, and X. A. K. Janse de Jonge, "The effects of different speed training protocols on sprint acceleration kinematics and muscle strength and power in field sport athletes," Journal of Strength and Conditioning Research, vol. 26, no. 6, pp. 1539-1550, 2012.

[14] R. G. Lockie, A. J. Murphy, M. D. Jeffriess, and S. J. Callaghan, "Step kinematic predictors of short sprint performance in field sport athletes," Serbian Journal of Sports Sciences, vol. 7, no. 2, pp. 71-77, 2013.

[15] R. G. Lockie, A. J. Murphy, A. B. Schultz, M. D. Jeffriess, and S. J. Callaghan, "Influence of sprint acceleration stance kinetics on velocity and step kinematics in field sport athletes," Journal of Strength and Conditioning Research, vol. 27, no. 9, pp. 24942503, 2013.

[16] C. D. Spinks, A. J. Murphy, W. L. Spinks, and R. G. Lockie, “The effects of resisted sprint training on acceleration performance and kinematics in soccer, rugby union, and Australian football players," Journal of Strength and Conditioning Research, vol. 21, no. 1, pp. 77-85, 2007.

[17] P. S. Maulder, E. J. Bradshaw, and J. W. L. Keogh, "Kinematic alterations due to different loading schemes in early acceleration sprint performance from starting blocks," Journal of Strength and Conditioning Research, vol. 22, no. 6, pp. 1992-2002, 2008.
[18] S. Nimphius, M. R. McGuigan, and R. U. Newton, "Relationship between strength, power, speed, and change of direction performance of female softball players," Journal of Strength and Conditioning Research, vol. 24, no. 4, pp. 885-895, 2010.

[19] S. Debaere, C. Delecluse, D. Aerenhouts, F. Hagman, and I. Jonkers, "From block clearance to sprint running: characteristics underlying an effective transition," Journal of Sports Sciences, vol. 31, no. 2, pp. 137-149, 2013.

[20] W. B. Young, R. James, and I. Montgomery, "Is muscle power related to running speed with changes of direction?" Journal of Sports Medicine and Physical Fitness, vol. 42, no. 3, pp. 282-288, 2002.

[21] R. Mann and J. Herman, "Kinematic analysis of Olympic sprint performance: men's 200 meters," Journal of Applied Biomechanics, vol. 1, no. 3, pp. 151-162, 1985.

[22] J. Slawinski, A. Bonnefoy, G. Ontanon et al., "Segmentinteraction in sprint start: analysis of 3D angular velocity and kinetic energy in elite sprinters," Journal of Biomechanics, vol. 43, no. 8, pp. 1494-1502, 2010.

[23] A. Struzik, A. Rokita, B. Pietraszewski, and M. Popowczak, "Accuracy of replicating static torque and its effect on shooting accuracy in young basketball players," Human Movement, vol. 15, no. 4, pp. 216-220, 2014.

[24] J. B. Krell and D. J. Stefanyshyn, “The relationship between extension of the metatarsophalangeal joint and sprint time for 100 m olympic athletes," Journal of Sports Sciences, vol. 24, no. 2, pp. 175-180, 2006.

[25] N. W. Ansari, Y. Paul, and K. Sharma, "Kinematic analysis of competitive sprinting," African Journal for Physical, Health Education, Recreation and Dance, vol. 18, no. 4, pp. 662-671, 2012.

[26] C. Ranjan, "The relationship of selected kinematical variables to the performance of runners in sprint start," International Journal of Sports Sciences and Fitness, vol. 1, no. 1, pp. 60-67, 2011.

[27] R. G. Lockie, S. J. Callaghan, and M. D. Jeffriess, "Acceleration kinematics in cricketers: implications for performance in the field," Journal of Sports Science and Medicine, vol. 13, no. 1, pp. 128-136, 2014.

[28] W. Young, S. Cormack, and M. Crichton, "Which jump variables should be used to assess explosive leg muscle function?" International Journal of Sports Physiology and Performance, vol. 6, no. 1, pp. 51-57, 2011.

[29] A. Struzik, G. Konieczny, K. Grzesik, M. Stawarz, S. Winiarski, and A. Rokita, "Relationship between lower limbs kinematic variables and effectiveness of sprint during maximum velocity phase," Acta of Bioengineering and Biomechanics, vol. 17, no. 4, pp. 131-138, 2015.

[30] A. A. Biewener, C. T. Farley, T. J. Roberts, and M. Temaner, "Muscle mechanical advantage of human walking and running: implications for energy cost," Journal of Applied Physiology, vol. 97, no. 6, pp. 2266-2274, 2004. 


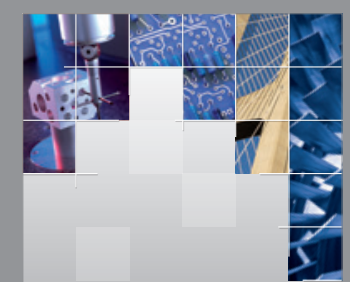

\section{Enfincering}
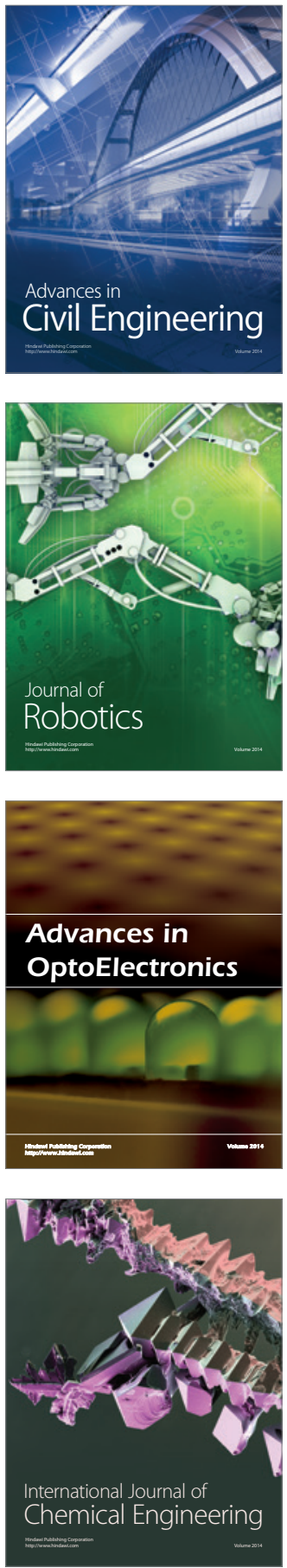

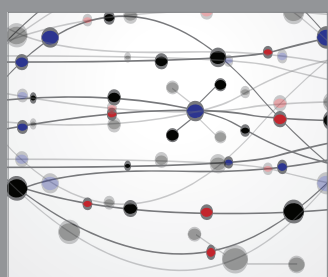

The Scientific World Journal

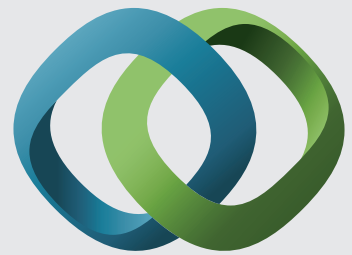

\section{Hindawi}

Submit your manuscripts at

http://www.hindawi.com
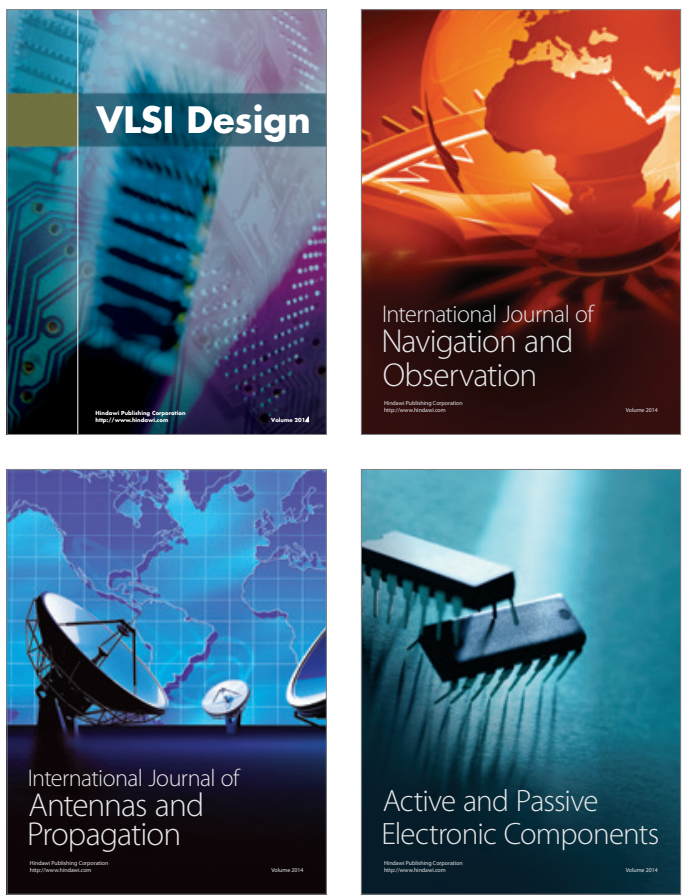
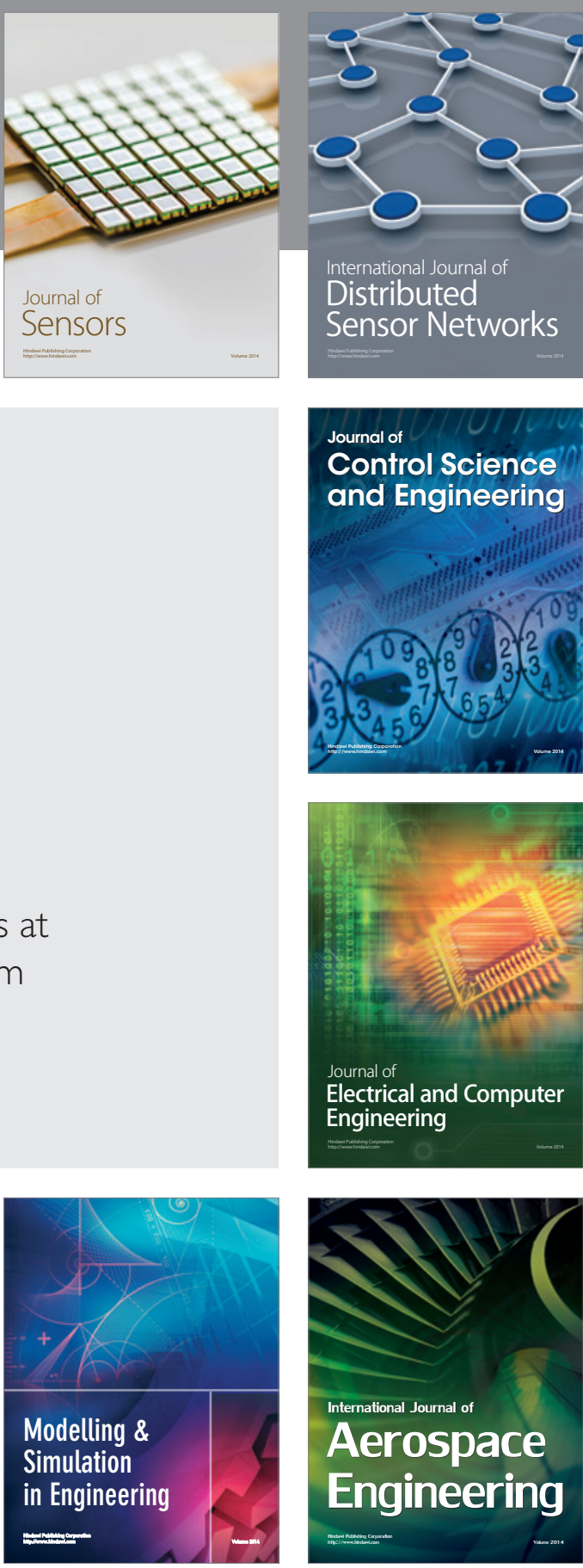

International Journal of

Distributed

Sensor Networks

Journal of

Control Science

and Engineering
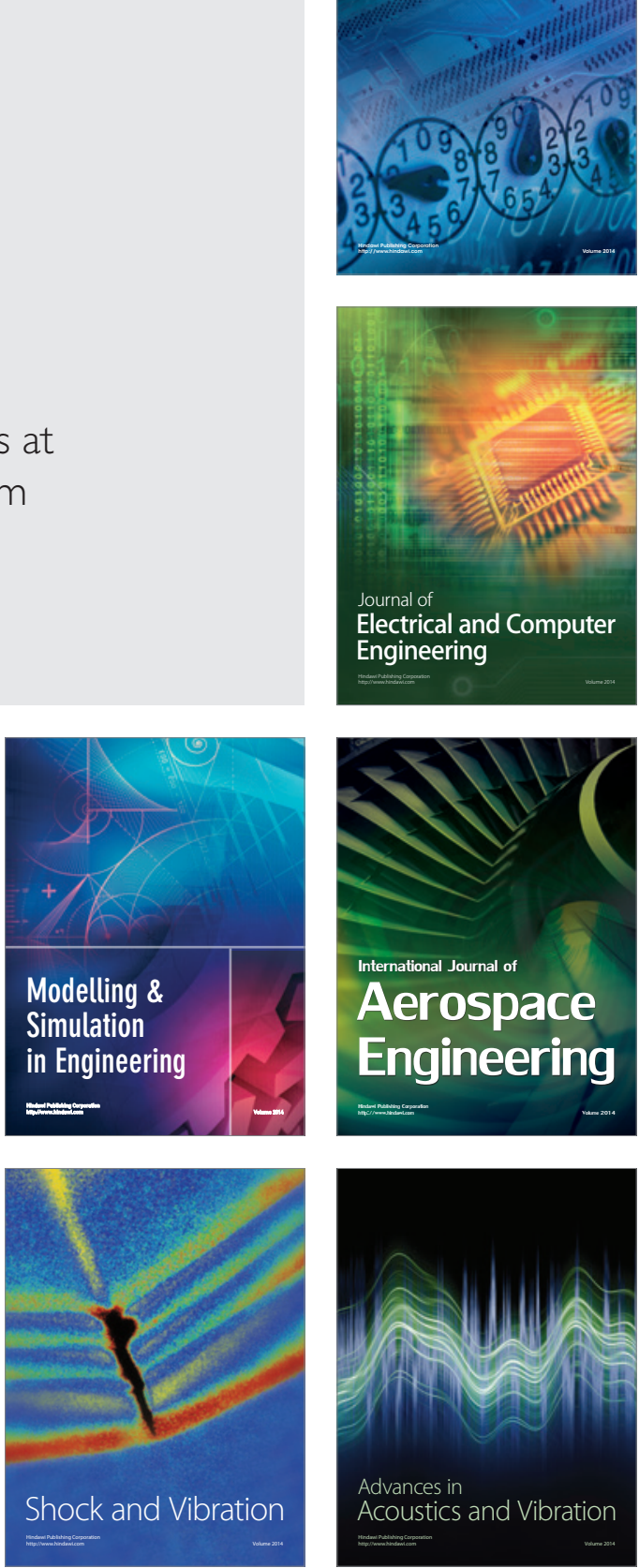